\title{
Información y conocimiento en organizaciones complejas
}

Information and knowledge in complex organizations

\author{
Marta Valentim \\ Universidade Estadual Paulista (UNESP/Marília) \\ valentim@valentim.pro.br
}

\begin{abstract}
Resumen
Las organizaciones vivencian una época muy competitiva, consecuencia de la economía globalizada, de los países que actúan en conjunto a partir de regiones geográficas, de consumidores más exigentes, en fin, comoresultado de una serie de circunstancias que, de alguna manera, interfieren en sus modelos de gestión y producción. Comprender la organización en su complejidad redimensiona el papel de la información y del conocimiento en ese ámbito. La información y el conocimiento tienen un papel fundamental en el ambiente corporativo, porque todas las actividades desarrolladas, desde el planeamiento hasta la ejecución de las acciones planeadas, así como el proceso decisorio, son apoyadas por información y conocimiento. La información y el conocimiento, insumos del hacer organizacional necesitan ser mejor gestionados. Para eso, los gestores precisan comprender desde el macroambiente hasta los microambientes informacionales-coginitivos que componen la organización e interactúan en conjunto con la sociedad; y precisan percibir los complejos contextos - político, económico, social, tecnológico y cultural-que rodean a las organizaciones en el mundo actual. Estudiar la información y el conocimiento, así como estudiar la gestión de la información y la gestión del conocimiento en organizaciones complejas se hace necesario, toda vez que son las personas, por medio de sus acciones/actividades, las que desarrollan las organizaciones haciéndolas competitivas y, así, posibilitan el desarrollo social y económico del entorno.
\end{abstract}

Palabras clave: Organizaciones del conocimiento. Gestión de la información. Gestión del conocimiento. Organizaciones complejas. Información y conocimiento.

\section{Introducción}

Es muy difícil discutir lo económico sin traer los asuntos políticos y sociales. No es que sea imposible, pero en el contexto de la Sociedad de la Información, sería extremadamente difícil.

Entiendo a la Sociedad de la Información como aquella económicamente cimentada en la información y el conocimiento y en las tecnologías

\begin{abstract}
The organizations are experiencing an extremely competitive epoch, consequence of the globalize economy, of the countries acting in partnership from geographical regions, of more demanding consumers, finally, as a result of a series of circumstances that, of some way, interfere in his models of management and production. Understanding the organization in its complexity re-dimensions the function of information and knowledge in that scope. Information and knowledge have a fundamental function in corporate environments, because all the activities-developed from their planning up to the execution of the planned actions, as well as all the decisive processes-are supported by information and knowledge. Information and knowledge-inputs of the organizational activity-are to be better managed. For that, the agents are to understand the macro and micro environments of information/knowledge that form the organization and interact with the society; and, as well, are to perceive the complex contexts-political, economic, social, technological and cultural-, that surround the organizations in the present world. Studying information and knowledge and its management in complex organizations is absolutely necessary, since the persons are, by means of his actions/activities, who develop organizations, doing them more competitive and, so, enabling the social and economic development of the their environment.
\end{abstract}

Keywords: Knowledge organizations. Information Management. Knowledge Management. Complex organizations. Information and knowledge.

de información y comunicación (TIC's). La información es la materia prima, el insumo básico del proceso; las tecnologías de información y comunicación son las estructuras que hacen posible el acceso, el almacenaje, el procesamiento y la diseminación del conocimiento, y el resultado del proceso (Valentim, 2002).

Primeramente, es importante comprender la organización como núcleo de la sociedad, en el 
sentido de que ella congrega personas. Para Morin (2005) la "empresa es colocada en un medio ambiente externo que a su vez integra un sistema eco organizado o ecosistema". En ese escenario, las organizaciones, sean públicas o privadas, son núcleos de esa sociedad, por cuanto sustentan la economía, generan empleos, profesionalizan y especializan la formación y actuación de los individuos, en suma, influyen en la cultura y en la propia sociedad.

La relación entre el poder económico (las organizaciones) y el poder político (los gobiernos) es extremadamente fuerte, pues históricamente verificamos que esa relación está enlazada a tal punto que, muchas veces, no percibimos donde comienza uno y donde termina otro. La globalización trajo para los gobiernos una figura, que ya existía anteriormente, pero que se consolidó de tal forma con la globalización, que modificó el grado del poder gubernamental. Los gobiernos son, cada vez más, apoyados por grupos económicos, que definen, de cierto modo, el rumbo económico, político y social, tanto a nivel macro (país, bloque económico), como a nivel micro (municipio, estado).

Por lo tanto, la construcción de una sociedad recibe gran influencia de las organizaciones. En ese sentido, las organizaciones son de hecho núcleos sociales de extrema importancia, para cada uno de los segmentos que componen una determinada sociedad. Al mismo tiempo que la organización influye en el medio en la que está inserta, recibe la influencia del medio influenciado. Esa dinámica transforma, poco a poco, los principios, los valores, las creencias, los comportamientos, las actitudes, etc. El resultado de esas interacciones y de la interdependencia entre las organizaciones y el medio en el que actúan, puede ser considerado como el que sustenta la estructura de la sociedad, en este caso la Sociedad de la Información.

\section{Interacción sociedad-organizaciones}

Discutir la interacción entre la Sociedad de la Información y las organizaciones, así como el papel que ellas tienen en ese contexto es fundamental. Los fenómenos organizacionales son aquellos que, de cierta forma, se insertan en la sociedad, ya que los individuos participan de los dos contextos. Las políticas públicas son elaboradas a partir de un punto de vista, actualmente más económico/ideológico, anteriormente más ideológico. Ese hecho modifica la forma y el entendimiento de las cuestiones sociales.

La información es el insumo para que cualquiera se desarrolle en el ámbito académico o industrial. El desarrollo requiere de contenidos infor- mativos que, una vez, procesados y disponibles, proporcionen el desempeño eficiente. La generación de conocimiento solamente es posible cuando la información es interiorizada por el sujeto, propiciando, por medio del establecimiento de relaciones cognitivas, nuevo conocimiento, que puede ser aplicado.

La relación sistemática entre política y economía, genera o no beneficios sociales. La Sociedad de la Información, por lo tanto, es resultado de esa relación. Cabe a los profesionales que estudian los fenómenos de esa sociedad, indicar a las organizaciones, la necesidad de invertir en recursos humanos capacitados e infraestructura, de modo que la Sociedad de la información sea de hecho una realidad y no apenas un plan o programa de acción gubernamental.

Por lo expuesto, considero que la Sociedad de la Información necesita urgentemente de la comprensión de su importancia, por parte de las organizaciones, pues solamente ellas pueden modificar los valores $y$, en consecuencia, la cultura de un país, que depende del acceso, generación, uso e intercambio de la información y conocimiento. Un país sin información es un país sin conocimiento, y un país sin conocimiento es un país sin desarrollo económico y social.

El papel del estado y de las empresas en el contexto de la Sociedad de la Información se modifica, ya que el estado ha reducido significativamente su presencia en el sector productivo $y$, por otro lado, las empresas se han preocupado de su responsabilidad social. Esa inversión de papeles le da un nuevo significado a la dimensión de la influencia de las organizaciones en la sociedad. Sainsaulieu e Kirschner (2006) consideran que las organizaciones influyen sobre las representaciones y estructuras sociales, como por ejemplo, en las relaciones de poder.

La historia de las organizaciones acompaña la historia de las tecnologías de información y comunicación y, en muchos momentos, esas historias se interrelacionan, puesto que las tecnologías modifican las actividades y rutinas de trabajo, exigen nuevas competencias para actuar, influyen en la creación de nuevas calificaciones que no existían anteriormente. En ese sentido, la Sociedad de la Información es expresión de un modelo político-ideológico, pero también, de un modelo organizacional y tecnológico.

$\mathrm{Si}$ es verdad que la Sociedad de la Información es aquella económicamente cimentada en la información, en el conocimiento y en las tecnologías de información y comunicación (TIC's) (Valentim, 2002) y, si por otro lado, las organizaciones ayudan a construir ese modelo de sociedad, puedo inferir que los elementos constitutivos de 
la Sociedad de la Información son los elementos constitutivos de las organizaciones.

Partiendo de ese presupuesto, considero que la información y el conocimiento poseen real importancia para las organizaciones contemporáneas toda vez que, a partir de la gestión de los procesos de información y de conocimiento es posible (Choo, 2003):

- Adaptarse a los cambios del ambiente en el momento adecuado y de manera eficaz.

- Empeñarse en el aprendizaje constante, el que incluye desaprender presupuestos, normas y creencias que perdieron validad.

- Movilizar el conocimiento y la experiencia de sus miembros para generar innovación y creatividad.

- Focalizar su conocimiento en acciones racionales y decisivas.

Comprender que esos elementos se constituyen en la base para diferentes acciones: toma de decisiones, planeamiento, estrategias, etc., que resultarán en el desarrollo de la organización, es el primer paso para desarrollar la percepción correcta de la relación e interdependencia existente. La interdependencia entre empresa (sociedad interna) con otros tipos de instituciones (sociedad externa) —como, por ejemplo, educacionales, filantrópicas, religiosas, asociativas, profesionales- es real, ya que esos diferentes contextos ambientales son visitados por los actores que en ella actúan, y por lo tanto, reciben toda la influencia cultural de esas "sociedades", trasladándola al ambiente organizacional (Figura 1).

Las organizaciones sufren presiones y al mismo tiempo presionan los diferentes ambientes sociales, la Sociedad de la Información, es un ejemplo de eso. No obstante, no hay duda, en cuanto al entendimiento de que las organizaciones son una forma de sociabilidad producida, transmitida, compartida y renovada (Sainsaulieu; Kirschner, 2006).

Defiendo, así, que la Sociedad de la Información recibe como la mayor fuerza influyente no sólo las tecnologías de información y comunicación, sino, también, las organizaciones y sus propias necesidades de evolución.

Las organizaciones son por lo tanto, grandes influyentes de ese modelo de sociedad, por cuanto las tecnologías de información y comunicación son, de hecho, resultado de esas influencias, ya que las organizaciones como organismos sociales productoras de sociabilidad y de identidades específicas en el seno de la sociedad (Sainsaulieu; Kirschner, 2006), es que intervienen a partir de esas representaciones en las lógicas político-económicas existentes.

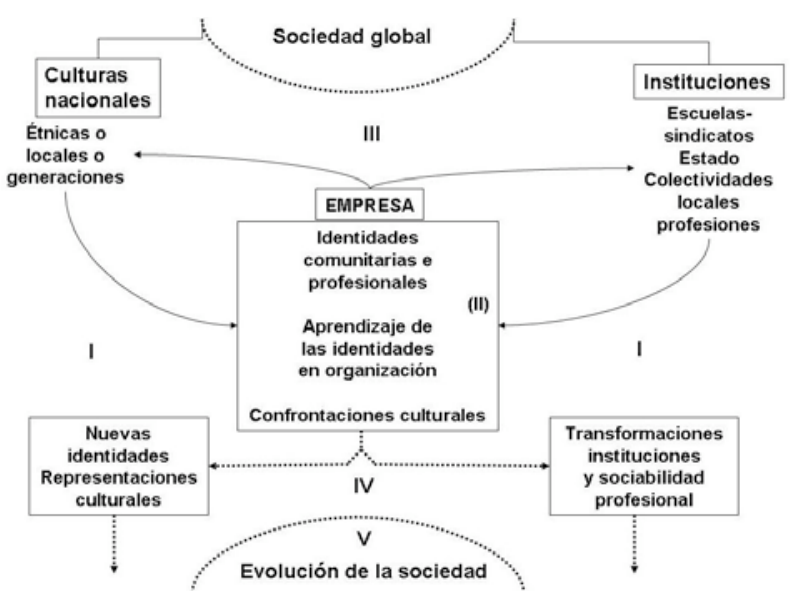

I: Transmisión de la cultura anterior

II: Secuencias de aprendizaje en el contacto de la experiencia

III: Reproducción de la sociedad

IV: Cambios en los valores y en las instituciones

V: Producción de la sociedad nueva

Figura 1. Relación e influencias: organizaciones y sociedad (Sainsaulieu, Kirschner, 2006, p. 263)

Las organizaciones son responsables de las interacciones y reacciones sociales (Morin y Moigne, 2000) de forma sistemática, y generan una dinámica sistémica. Esa dinámica es compleja, pues las envuelven y las influencian al mismo tiempo en que influyen y envuelven otras dimensiones.

La Sociedad de la Información representa una dimensión cultural compleja, puesto que ella está cimentada en la información y el conocimiento, cuya base es la cultura acumulada a través del tiempo, de la historia del propio hombre y de sus representaciones. A todo eso, se suman las tecnologías de información y comunicación, cuyo papel político integrador es real, pero que, al mismo tiempo, vivencian una paradoja desintegradora en relación a la economía

Para Morin (2005b) "la humanidad surge de una pluralidad y de una yuxtaposición de trinidades: la trinidad individuo / sociedad / especie. [...] no sólo los individuos están en la sociedad, la sociedad también está en los individuos, encurtiéndoles desde su nacimiento, su cultura". Si las organizaciones son las personas que en ella actúan, entonces puedo proponer una nueva trinidad: individuo-organización/sociedad.

La relación entre esos elementos es real y dinámica, pues cada uno influye en el otro. Por eso mismo, no podemos separar la organización de la sociedad ni viceversa, ya que el elemento común entre esos dos mundos es el mismo, sea cual fuese: el individuo. 


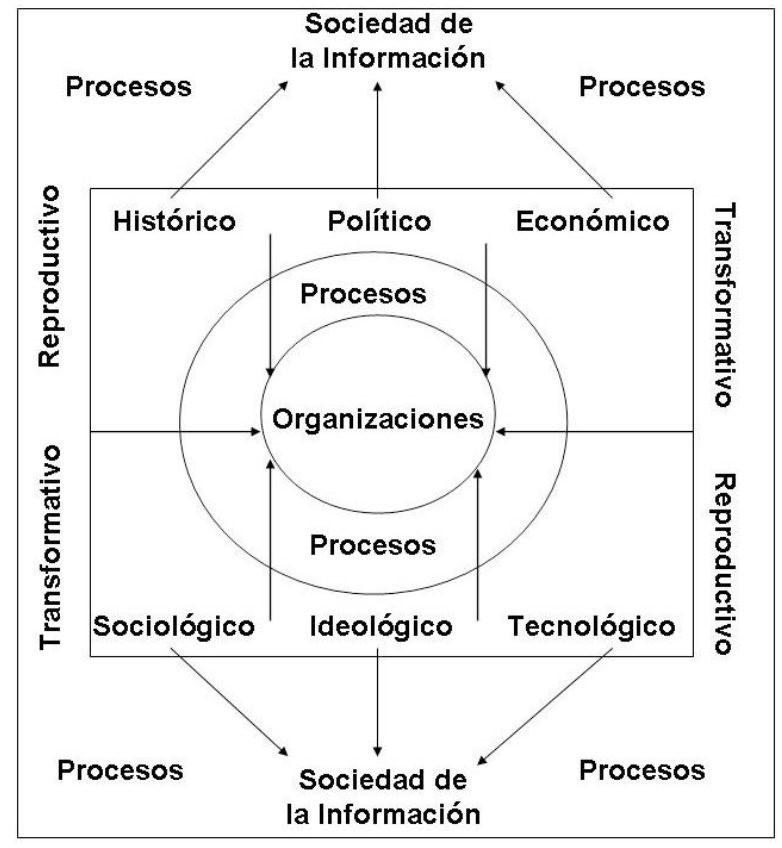

Figura 2. Dimensiones de la Sociedad de la Información

Los procesos históricos, políticos, económicos, sociológicos, ideológicos, tecnológicos, entre otros, pueden ser reproductivos o transformativos, y las organizaciones son el núcleo central de esos procesos, influyendo sobremanera tanto los tipos de procesos, como la calificación de esos procesos, esto es, reproductivos o transformativos. El hombre vive la mayor parte del tiempo en ambientes organizacionales. Por ese motivo, la influencia de las organizaciones es enormemente significativa.

Observo así, una relación directa y compleja entre las organizaciones y la sociedad. Su interpretación es real. Su interacción es real. La Sociedad de la Información depende del desarrollo organizacional, así como de las organizaciones dependen del desarrollo social, pues los actores son los mismos, por eso ese universo está constituido de cierta complejidad auto-ecore-organizadora (Morin; Moigne, 2000).

Considero que cada sociedad tiene su propia cultura. Aunque algunas de ellas sean similares, al analizarlas, percibimos sus especificidades. En el caso de la Sociedad de la Información, cuyo modelo está cimentado en principios y valores diseminados ampliamente por el planeta, ciertamente recibirá influencia de la cultura local. Eso demuestra que tenemos diferentes "Sociedades de la Información", a pesar de que ella ha surgido como una propuesta políticoeconómico-ideológica muy bien delineada. En ese contexto, esas "sociedades" son el resulta- do de las interacciones de la tríada propuesta: individuo-organización/sociedad.

\section{Información y conocimiento organizacional}

Los ambientes organizacionales complejos son apoyados por información y conocimiento, conforme ya fue mencionado anteriormente, lo que de hecho traduce la importancia de esos dos elementos para la vida organizacional. Morin (2005a) afirma que "la complejidad coincide con una parte de la incertidumbre, proveniente de los límites de nuestro entendimiento, o de aquella inscrita en los fenómenos. Sin embargo, la complejidad no se reduce a la incertidumbre, es la incertidumbre en el seno de lo sistemas ricamente organizados". Por eso mismo, destaco el papel de la información y del conocimiento en ambientes de esa naturaleza, cuya importancia resalta por amenizar o reducir la sensación de incertidumbre, que es inherente a los procesos humanos en ámbitos organizacionales.

Se considera que la información precede a la comunicación, la tecnología, el conocimiento y la acción (Ilharco, 2003). Así, la información es considerada insumo del saber y del hacer en diferentes contextos y diferentes objetivos. En el ambiente organizacional, esta afirmación se concretiza de forma contundente, ya que considero la información insumo del hacer organizacional, para cualquier sector, en cualquier especialidad, en cualquier segmento económico.

Las organizaciones en su complejidad requieren un continuum informativo, que apoye sus estrategias, sus acciones y la construcción de conocimiento corporativo. Siendo así, la información debe ser reconocida como elemento clave para el desarrollo organizacional. Es importante discutir el concepto de información, cuyas características, en mi opinión, son claras y son ampliamente discutidas por diferentes autores de diferentes áreas del conocimiento como Auckland (1991), Wurman (1995), Davenport e Prusak (1998), Morin (1999), Choo (2003), Zins (2007) entre otros autores. La información es, al mismo tiempo, objeto y fenómeno, puesto que puede ser destacada y analizada por si misma y, también, puede ser parte de un proceso. Considero que solamente podemos nombrarla "información", si la comprendemos, esto es, si existe por parte del sujeto cognitivo consenso en relación a su significado; en caso contrario, no es información. Otro aspecto que considero importante se refiere a las cualidades relevancia y propósito inherentes al término "información", ya que el sujeto cognitivo busca la información con determinado objetivo, sea conscientemente o in- 
conscientemente. Por eso mismo, la información está muy vinculada al sujeto, pues requiere la mediación humana: lo que es información para uno puede no ser información para otro. Así, el sujeto cognitivo replantea el significado de información, una vez que infiere síntesis y contexto en ella.

Además de todo eso, es importante rescatar la importancia de la información en cuanto a su papel en explicitar el conocimiento construido a lo largo de los tiempos. Cada sujeto cognitivo es responsable de la construcción del conocimiento, y ese mecanismo existe de manera natural. Sin embargo, solamente cuando el sujeto cognitivo explicita su conocimiento, por medio del lenguaje, aquí comprendido de forma lato, de hecho el conocimiento es consolidado, o sea, la información se hace parte del proceso de construcción del conocimiento: no existe construcción del conocimiento sin uso de la información de cualquier tipo/especie. Ese mecanismo dual es esencial para comprender la información en contextos organizacionales.

El conocimiento es producto de un sujeto cognitivo que a partir de la internalización de diferentes informaciones y percepciones elabora o reelabora su "nuevo" conocimiento. Creo que el conocimiento construido por un individuo alimenta la construcción del conocimiento colectivo; y, por otro lado, el conocimiento colectivo alimenta la construcción del conocimiento individual en ambientes organizacionales (Valentim, 2007, p. 20-21):

Por lo tanto, el conocimiento colectivo y el conocimiento individual, en el ámbito corporativo, son frutos de las interacciones, del sistema y de la propia organización. La complejidad del ambiente refleja en cada persona de modo único, el cual a su vez, construye significado a partir de la percepción individual, retirada de la percepción colectiva. Por eso, la construcción de conocimiento colectivo es resultado natural del conocimiento individual construido en el espacio corporativo. Las personas realizan interacciones -acción-clave para la construcción de conocimiento colectivo-, por medio del compartir y la socialización del conocimiento individual.

La información y el conocimiento son insumos del hacer organizacional y son fundamentales para el proceso decisorio. No consigo separar información y conocimiento, puesto que uno alimenta al otro, o sea, es un proceso dual necesario para la evolución del sujeto. En ese sentido, todo el trabajo realizado en el área de la Ciencia de la Información debería tomar en cuenta esa relación extremadamente fuerte e inseparable.

El mundo de la ciencia/científico tiene total percepción de la importancia de la información para el saber/hacer; en cambio, el mundo del trabajo no siempre reconoce esa importancia. Las organizaciones complejas, son entendidas como aquellas organizaciones responsables de la interacción y reacción sociales de forma sistemática. Esa dinámica es compleja, pues las envuelven y las influyen al mismo tiempo en que influyen otras dimensiones de la sociedad.

La información en organizaciones complejas no es obvia, no es transparente, no es comprendida por todos los sujetos que en ella actúan, justamente por las características mencionadas anteriormente. De hecho, cada sujeto, nombrará lo que para él es información. Exactamente, en ese aspecto es en el que reside la complejidad de la información, en ambientes extremadamente complejos. Por eso mismo, ese objeto (la información) y los fenómenos relacionados con él deben ser los temas de estudio, teniendo en cuenta el conocimiento de las problemáticas que lo envuelven.

La información puede ser considerada objeto, pues es a partir de una información explícita en algún soporte, que visualizamos el conocimiento construido por un individuo o grupo de individuos. Un informe solamente podrá existir a partir de la explicación del conocimiento de alguien o de un grupo. Por otro lado, la información también puede ser un fenómeno; esto es, el individuo o el grupo que elaboró el informe necesitó realizar un proceso cognitivo variable, después de realizar diferentes acciones cognitivas individuales y colectivas como, por ejemplo, relacionar, aislar, asociar, desasociar, analizar y sintetizar, obteniendo al final del proceso el conocimiento explícito, en el formato del informe.

Como fenómeno la información puede ser emancipadora, ya que a partir de ella, el sujeto cognitivo o el grupo de sujetos cognitivos podrán realizar el proceso cognitivo individualmente o colectivamente, con más seguridad, confianza y tranquilidad. Teniendo la seguridad de que, al final del proceso, el conocimiento explícito en el formato del informe, posee un contenido confiable y consistente. Aún, como fenómeno la información puede propiciar la condición de poder, de subyugación, de diferencia, puesto que el sujeto cognitivo o el grupo de sujetos cognitivos que poseen informaciones relevantes podrán realizar procesos cognitivos más eficientes que los otros individuos o grupos que no tienen las informaciones relevantes a su alcance o disposición. Así, el conocimiento explícito, en el formato de informe, de aquellos que poseen las informaciones relevantes será mejor elaborado, que por aquellos que no poseen las informaciones relevantes a su alcance o disposición. 
De este modo, los fenómenos informativos pueden ser estudiados bajo diferentes perspectivas (Figura 3). En el caso de las organizaciones, los cuatro cuadrantes pueden ocurrir al mismo tiempo. Por eso, la complejidad y la necesidad de comprender esas perspectivas de forma más profunda en el ámbito de la Ciencia de la Información. La información, por lo tanto, es insumo organizacional, cuyas características son inherentes al ambiente, contexto y sujetos.

El uso de la información puede ser realizado a partir de esas diferentes perspectivas. La única seguridad es que el uso de la información disminuye la inseguridad y la incertidumbre del sujeto cognitivo y, consecuentemente, afecta el proceso decisivo y las estrategias y acciones organizacionales.

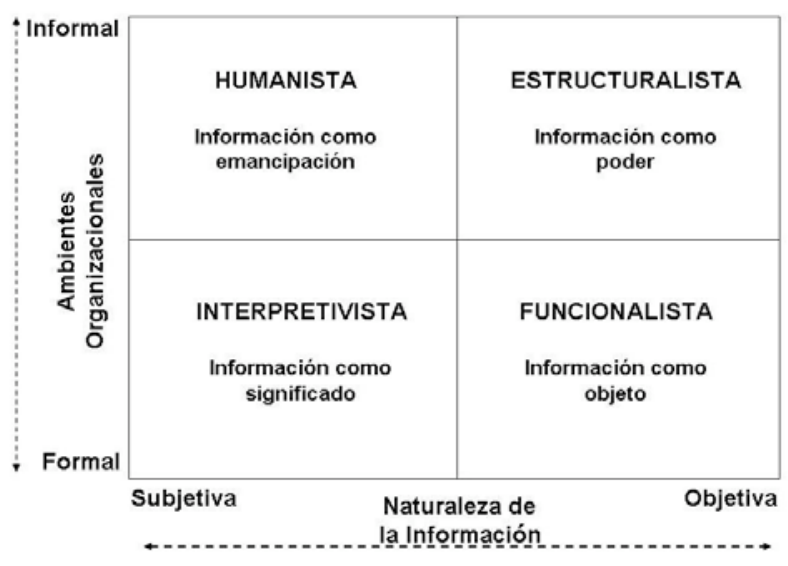

Figura 3. Naturaleza de la Información en Ambientes Organizacionales (Adaptado de ILHARCO, p.47)

Partimos del presupuesto que la búsqueda y el uso de información están relacionados a una acción, así sea inconsciente. Tal condición califica la información con un insumo extremadamente relevante para contextos complejos como las organizaciones.

Las organizaciones se desenvuelven a partir del aprendizaje organizacional, cuyos aspectos están relacionados con la información y al conocimiento, puesto que el aprendizaje se da probando y reconstruyendo la acción (Choo, 2003).

El aprendizaje organizacional, también, se vale de un proceso dual, pues al mismo tiempo en que relaciona, aísla; asocia, disocia; analiza, sintetiza. Esos movimientos están cimentados en información y conocimiento y es esa dinámica la que proporciona la acción (Morin, 1999).

Choo (2003) cree que las "organizaciones procesan y usan la información en tres distintas arenas: creación de significado, construcción de conocimiento y toma de decisión". En cada una de esas arenas esos movimientos de diálogo ocurren y, para eso, es necesario que el sujeto cognitivo realice la acción de mediarlas.

Se destaca que nadie en el ámbito organizacional posee todas las competencias necesarias para hacer el trabajo de la organización como un todo (Gardner et al., 1998). Es necesario que diferentes personas, con diferentes competencias, y a partir de la interacción de esas competencias cumplan los quehaceres de una determinada organización. Por eso mismo, la información y el conocimiento son importantes para los ambientes de esa naturaleza.

\section{Consideraciones finales}

El acceso y el uso de informaciones en ambientes organizacionales puede contribuir a amenizar diferentes aspectos que afectan al desarrollo de la organización como, por ejemplo, el aprendizaje organizacional, las prácticas organizacionales, la creatividad innovadora, y el grado de incertidumbre en el proceso de decisión, entre otros aspectos que influyen el día a día de las organizaciones.

Además de eso, contrariamente a lo que diferentes investigadores afirman, las tecnologías de la información y comunicación se pueden tornar problemáticas en la medida en que se tornan indistintos los papeles históricos de los gerentes como pensadores, y de los operarios como ejecutores (Zuboff, 1988). De hecho, el uso de las tecnologías de información y comunicación en la Sociedad de la Información es una realidad que trasciende el ámbito organizacional. Este contexto exige de las personas competencias específicas $y$, por lo tanto, presiona las estructuras conservadoras, puesto que modifica las relaciones e interacciones internas. La información, aquí, tiene un papel fundamental, pues a partir de ella, el individuo podrá asegurarse de que los movimientos de diálogo realizados son correctos o próximos a lo correcto.

El desempeño organizacional es directamente proporcional a la capacidad de diálogo de sus actores. Nuevamente, la información tiene un papel fundamental en esa dinámica, puesto que es insumo para que esa dinámica ocurra de forma satisfactoria. En ese sentido, gerenciar las informaciones, con el objetivo de apoyar las acciones organizacionales, se hace necesario.

La gestión de la información y la gestión del conocimiento son entendidas como acciones complementarias, ya que la gestión de la información "actúa directamente sobre los flujos 
formales de la organización; su foco es el negocio de la organización y su acción está restringida a las informaciones consolidadas en algún tipo de soporte (impreso, electrónico, digital, etc.) o sea, lo que está explícito", y la gestión del conocimiento "actúa directamente sobre los flujos informales de la organización, su foco es el capital intelectual corporativo y su acción está restringida a la cultura y la comunicación corporativa, o sea, lo que no está explícito. En la literatura, se utiliza de forma recurrente la expresión conocimiento tácito" (Valentim, 2007).

En ese sentido, la gestión de la información y la gestión del conocimiento actúan con el objeto de disminuir las situaciones ambiguas y con alto índice de incertidumbre, posibilitando a las personas de la organización el acceso y el uso de informaciones que repercutirán sobre esas situaciones. El nivel de complejidad de la situación requiere atención en cuanto a la precisión, relevancia y propósito de la información. El ambiente organizacional complejo necesita de condiciones que amenicen la complejidad de los procesos existentes en este ámbito y la información es un elemento que proporciona la disminución de esa complejidad.

Para eso, es necesario que las organizaciones posean un sector especializado que se encargue de la gestión, organización, tratamiento y diseminación de la información. El trabajo con la información requiere competencias específicas $y$, sobre este aspecto, el profesional de la información puede ayudar enormemente, puesto que propicia los quehaceres informativos que califican la información, así como la precisión, relevancia y propósito.

Las organizaciones complejas necesitan redimensionar el papel de la información y del conocimiento, dando un nuevo significado a su valor para los individuos que en ella actúan y para la propia organización.

\section{Referencias}

Buckland, M. (1991). Information as thing. // Journal of the American Society of Information Science. 42:5 (Jun. 1991) $351-360$

Choo, C. W. (2003). A organização do conhecimento: como as organizações usam a informação para criar significado, construir conhecimento e tomar decisões. São Paulo: SENAC, 2003. 425 p.

Davenport, T.; Prusak, L. (1998). Ecologia da informação: por que só a tecnologia não basta para o sucesso na era da informação. São Paulo: Futura, 1998. 316p.

Gardner, H. et al. (1998). Inteligência: múltiplas perspectivas. Porto Alegre: Artmed, 1998. 360p.

Ilharco, F. (2003). Filosofia da informação: uma introdução à informação como fundação da acção, da comunicação e da decisão. Lisboa: Universidade Católica Editora, 2003. 207p.

Morin, E. (2005a). Introdução ao pensamento complexo. Porto Alegre: Sulina, 2005(a). 120p.

Morin, E. (1999). O método 3: o conhecimento do conhecimento. Porto Alegre: Sulina, 1999. 288p.

Morin, E. (2005b). O método 5: a humanidade da humanidade. 3.ed. Porto Alegre: Sulina, 2005(b). 312p.

Morin, E.; Moigne, J.-L. L. (2000). A inteligência da complexidade. 3.ed. Uberaba: Peirópolis, 2000. 268p.

Sainsaulieu, R.; Kirschner, A. M. (2006). Sociologia da empresa: organização, poder, cultura e desenvolvimento no Brasil. Rio de Janeiro: DP\&A, 2006. 464p.

Valentim, M. L. P. (Org.) (2007). Informação, conhecimento e inteligência organizacional. 2.ed. Marília: FUNDEPE, 2007. 278p.

Valentim, M. L. P. (2002). Inteligência competitiva em organizações: dado, informação e conhecimento. // Datagramazero. 3:4 (Ago. 2002) 1-13. http://www. dgz.org.br/ago02/Art_02.htm (2007-05-23)

Wurman, R. S. (1995). Ansiedade de informação: como transformar informação em compreensão. 5.ed. São Paulo: Cultura Editores, 1995. 380p.

Zins, C. (2007). Conceptual approaches for defining data, information, and knowledge. // Journal of the American Society for Information Science and Technology. 58:4 (Feb. 2007) 479-493.

Zuboff, S. (1988). In the age of the smart machine: the future of work and power. New York: Basic Books, 1988. 468 p. 